\title{
AN APPLICATION OF COMPUTER VISION SYSTEMS TO SOLVE THE PROBLEM OF UNMANNED AERIAL VEHICLE CONTROL
}

\author{
Alexey Y. Aksenov ${ }^{1}$, Sergey V. Kuleshov ${ }^{2}$, Alexandra A. Zaytseva ${ }^{3}$ \\ Saint-Petersburg Institute for Informatics and Automation of RAS \\ $14^{\text {th }}$ line V.O., 39, Saint-Petersburg, 199178, Russia \\ Phone: +7 812 3235139.E-mails: ${ }_{3}^{1}$ _aksenov@mail.iias.spb.su, ${ }^{2}$ kuleshov@iias.spb.su, \\ 33cher@iias.spb.su
}

\begin{abstract}
The paper considers an approach for application of computer vision systems to solve the problem of unmanned aerial vehicle control. The processing of images obtained through onboard camera is required for absolute positioning of aerial platform (automatic landing and take-off, hovering etc.) used image processing on-board camera. The proposed method combines the advantages of existing systems and gives the ability to perform hovering over a given point, the exact take-off and landing. The limitations of implemented methods are determined and the algorithm is proposed to combine them in order to improve the efficiency.

Keywords: computer vision, unmanned aerial vehicle control
\end{abstract}

\section{Introduction}

The rapid development of technology of autonomous robotic systems and the need of unmanned aircraft in various fields, including military purposes, led to the accelerated pace of development and deployment of small unmanned (UAVs) and remotely piloted (RPV) aircraft. The most actively developing are the multi motor flying platforms (tri-, quad-, hexa-copters).

The common features for all objects of given class are the design and the principle of flight. The central part of multicopter is intended to carry the equipment, cargo and battery. Radially from the centre the micro engines with rotors are mounted on beams, forming star-shaped layout of copter. Nevertheless such a symmetrical layout assumes presence of front and rear parts considering the direction of flight. During the flight the multicopter maintains relative to ground horizontal position, move sideways, change altitude and hover. In presence of additional equipment the automatic and half-automatic flights are possible. To compensate the twist due to momentum the rotors rotate in different directions. To perform a movement the quadrocopter is needed to be taken out of balance by throttling combinations of rotors. As a result quadrocopter tilts and starts to fly in needed direction. To rotate quadrocopter clockwise the front and rear rotors are spinning-up and left and right rotors are slowing down. The counter clockwise twist is done by analogy (Schmid, 2012).

Multi motor UAVs have several advantages compared to other unmanned and manned aircraft, especially the ability to survey small ground objects with high spatial resolution. Thus multi motor UAV unlike helicopter are more stable in air, less expensive to maintain and easier to control (Barbasov, 2012).

In general multi motor UAV is a universal, efficient and simple type of unmanned vehicle, which can take advantage over traditional helicopter design on the market and become useful instrument for mass media, photo and video industry etc. The greatest interest in UAVs show government agencies and services, which functions are related to the protection, control and monitoring of the facilities, including the liquidation of emergency situations, energy companies, and companies whose activities are related to obtain spatial data (Zinchenko, 2012).

Increasing the autonomy of UAVs and the transition to a fully autonomous UAV-robots working on the basis of the flight plan are made by improving the electronic autopilot and stabilization systems.

Among the existing stabilization systems for which information is not closed, the NAZA system (DJI Innovations, 2013) should be noted - a light, multi-axis control system designed specifically for small aircraft and including a flight controller, gyroscopes, accelerometers and barometer.

The other systems such as Wookong Multi-Rotor stabilization controller and FY-DoS GPS (flight control system for multi rotor LA for commercial and industrial autopilot applications) are belonging to the class of inertial stabilizing systems with hovering and auto-return functions. 
All of the mentioned above stabilization systems unsatisfactorily solve a number of specific operations that are relevant for flying robots:

- auto take-off;

- auto landing at given site; of time.

- automatic maintaining of absolute spatial position at low altitudes (hovering) for a long period

The most urgent and difficult problem to solve is a problem of hovering over a given point, as it is most prolonged mode of stabilization which provokes a big deviation from the given spatial point caused by the wind conditions and the drift of the inertial navigation system parameters. Therefore hovering is an advisable check for the developed method of using video data from on-board cameras to stabilize aircraft.

At present the following methods to solve the problem of hovering over the given point are exist:

1. The usage of external markers. Advantages - accuracy and ease of implementation in a limited space (Figure 1). Disadvantages - requires preliminary preparation of area, which achievable only in limited areas.

2. The usage of barometric sensors and inertial navigation systems. Advantages - versatility. Disadvantages - parameters drift, poor resistance to the wind.

3. The usage of satellite navigation GPS / GLONASS. Advantages - simplicity and versatility of the method. Disadvantages - large error, the inability to solve the problem for small heights and areas.

4. The usage of cameras and video processing techniques (Computer Vision).

The second and third methods in its current form are fundamentally inapplicable to resolve problem at low altitudes. The first and fourth methods are potentially useful for implementing, but with certain limitations.

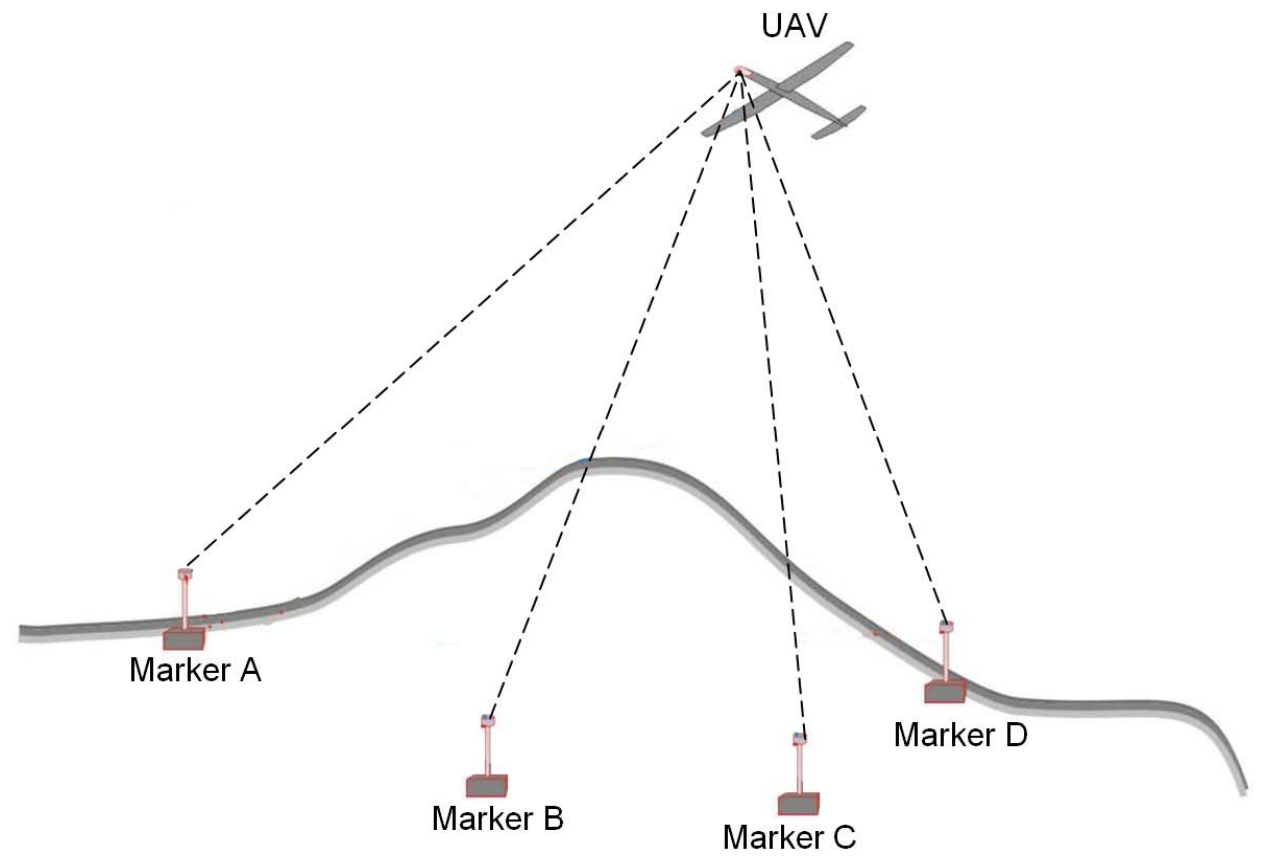

Figure 1. The usage of external markers

The typical accuracy of modern GPS receivers in horizontal plane reaches 6-8 meters given good satellite visibility and correction algorithms. Over the territory of USA, Canada and other countries it is possible to increase accuracy to 1-2 meters using the differential mode. Thus the use of GPS system over other territories for this issue appears to be not efficient.

As a variant of application that utilizes both markers and computer vision it is worth to mention a multicopter collective control project developed by Institute for Dynamic Systems and Control (IDSC) at ETH Zurich (ETH IDSC, 2014). The computer vision system with two or four cameras is used to automatically maintain the given direction of flight. This system achieves high accuracy but needs preliminary prepared workspace because the markers are located on UAV and the computer vision system is located stationary outside the UAV (Ritz, 2012).

In this paper an attempt is made on hardware and software solution for the problem of hovering over a given point at a low altitude for a long time by combining the existing methods without the use of external markers. 


\section{Algorithm of Motion Compensation Based on Computer Vision}

To solve the problem hovering at a given point (the stabilization of the spatial position of the aircraft by the images from the cameras) the analysis of local motion vectors blocks is proposed (VirtualDubDeshaker filter, available in works by Vatolin, Soldatov, Strelnikov, 2011 and Thalin, 2011).

The proposed method tries to find the panning, rotation and zoom combination that, when applied to the current image, makes it similar the previous image. This procedure takes blocks of pixels in the two images and finds the shift that makes them match with least error. The first step determines the motion of the whole image. This is accomplished by reducing the scale of images. The next step is similar to first but it compares images in a scale twice as big and with four times as many blocks. This step is repeated until the motions for all blocks are determined (Figure 2). The last step finds the best values for panning, rotation and zoom based on these motion vectors which are then transmitted to aircraft autopilot control system (Figure 3).

Videosequence

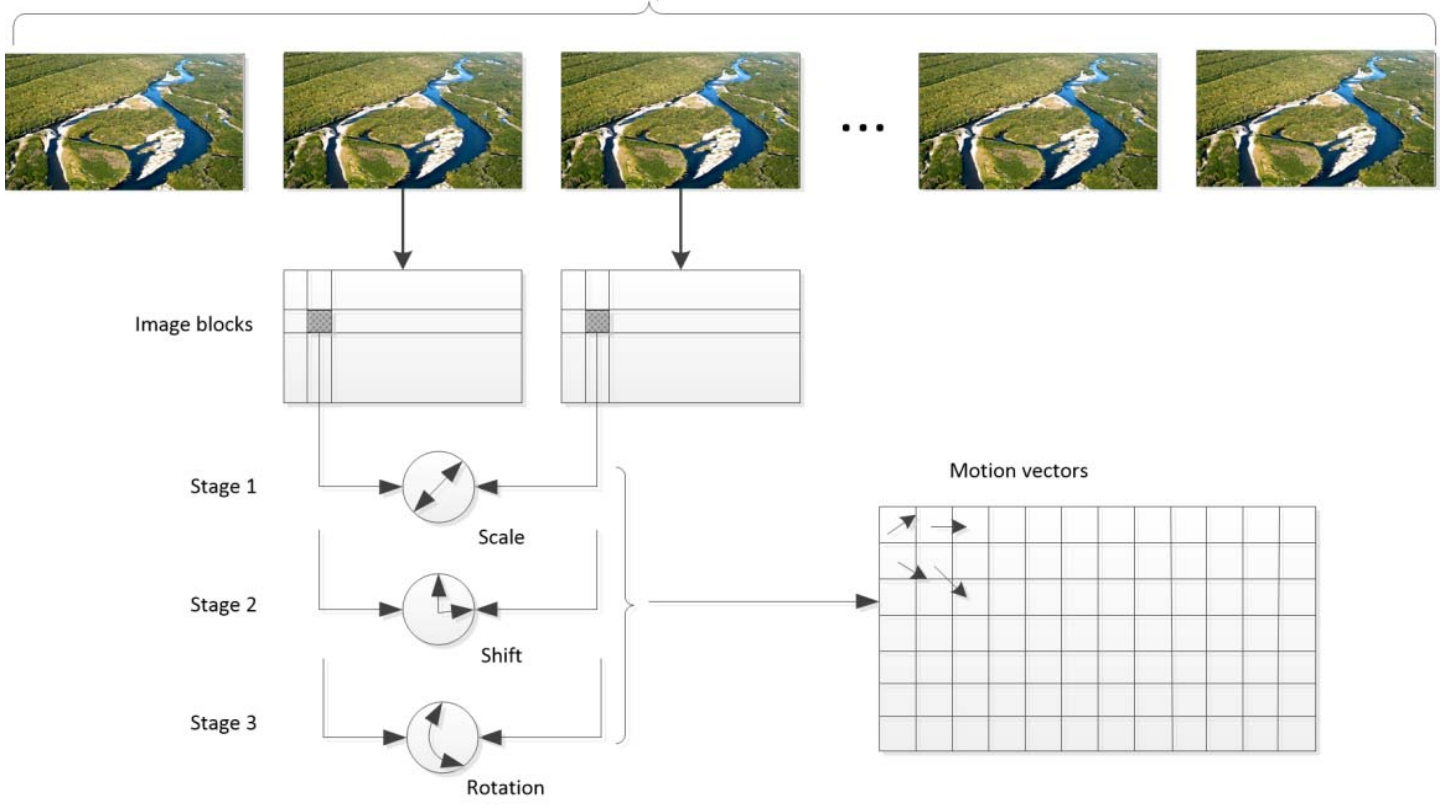

Figure 2. Algorithm of motion vector's determination

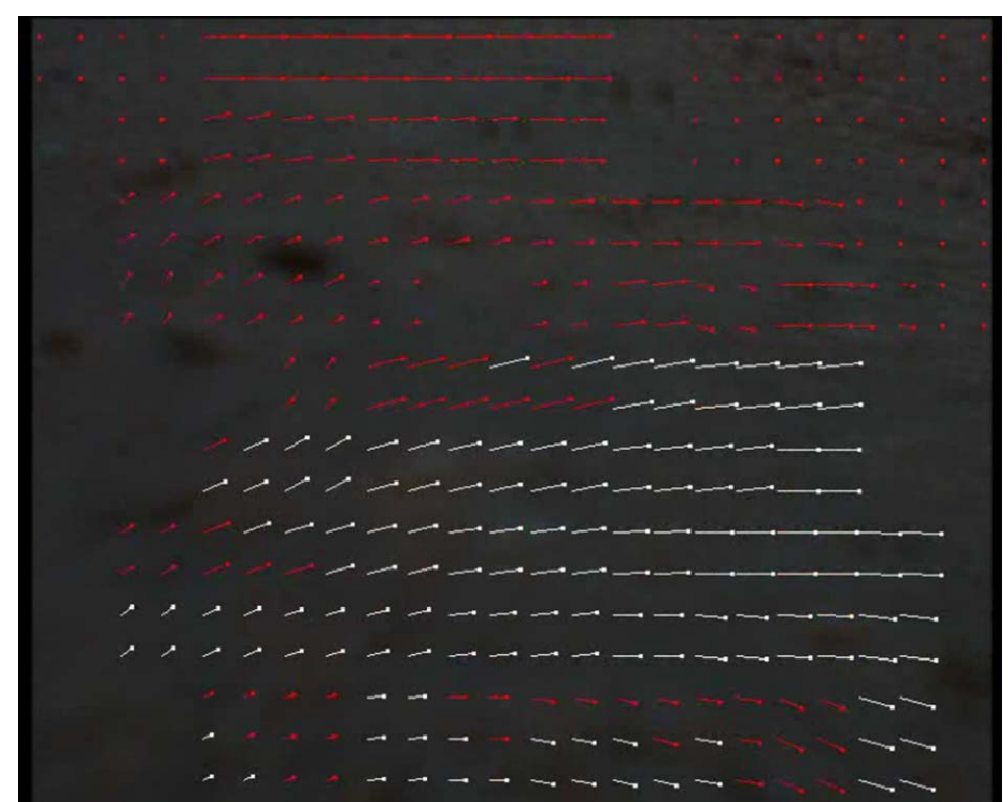

Figure 3. The result of motion estimation step 
Using the results of onboard camera images analysis and computation of the camera motion relative to horizontal surface fragment, the control impulses for flying platform engines necessary to maintain given spatial position are forming. To eliminate the influence of tilt (roll and pitch values) in the processing the instantaneous values of the angle obtained from an inertial navigation system, in particular a gyroscope are used.

The proposed algorithm has a limited usage in conditions of low contrast low sharpness image of a horizontal surface, which may be due to the properties of the surface (i.e. water), limited visibility associated with weather conditions, as well as to the lack of surface lighting.

\section{System Realization}

The electronic control unit for an autonomous UAV is proposed to implement as a multiprocessor system, implemented in the form of a "system on a chip" (SoC). For reliability the automatic control system (ACS), which forms the controlling influences on the servo motors, is separated from the processor that analyzes images from onboard cameras and performing flight mission (Figure 4).

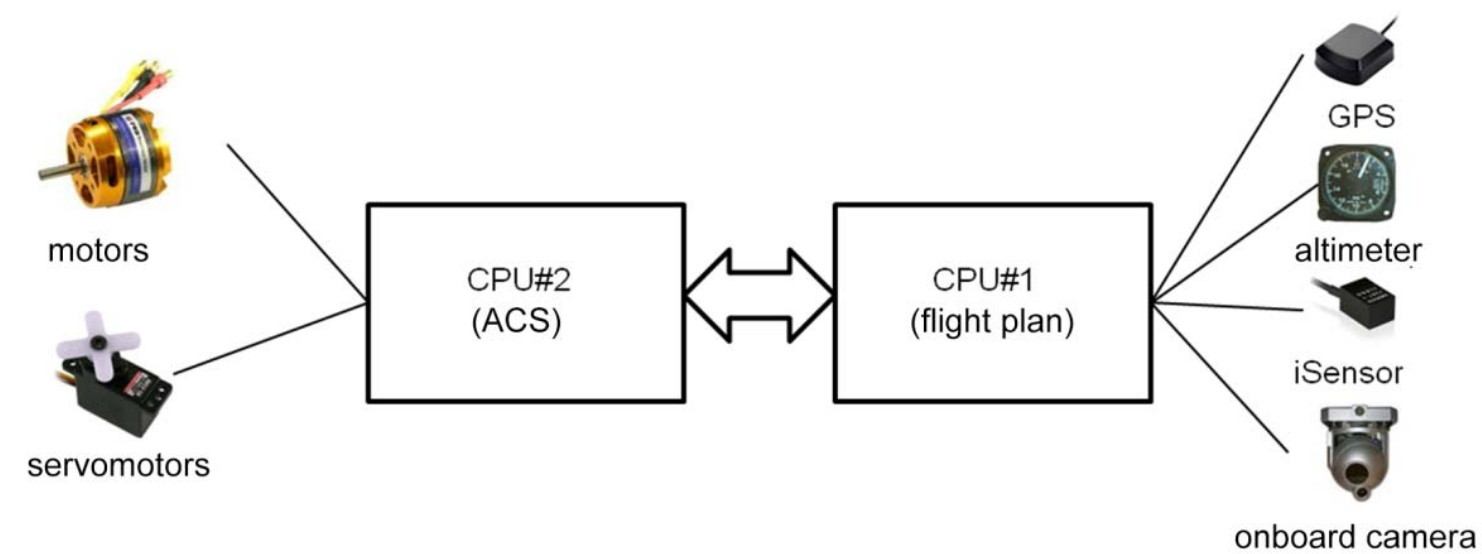

Figure 4. The structure of software-hardware components interaction

Physically, both processors are implemented on FPGA Altera, which also comprises peripheral memory controllers, interfaces for interfacing with the radio module and decoder (Figure 5).

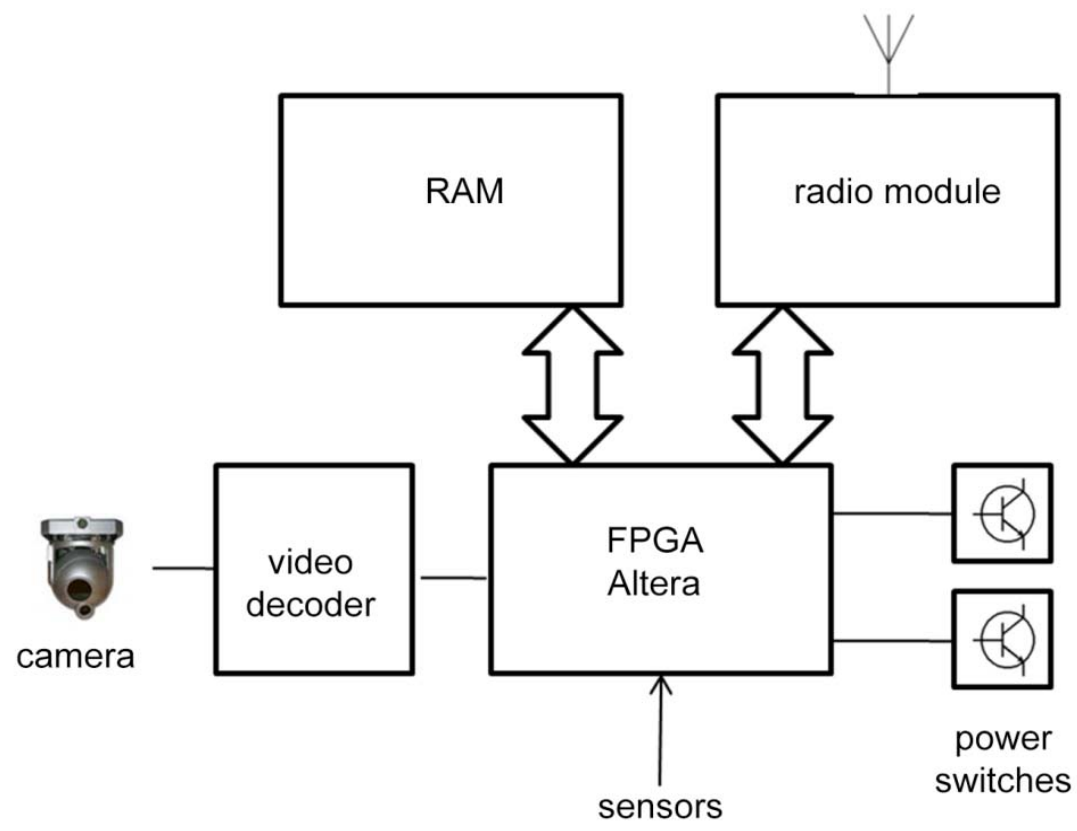

Figure 5. Structural schematics 
The peculiarity of the use of GPS systems is the presence of errors, resulting in the emergence of coordinate errors and, consequently, the spontaneous movement of the UAV near the point of equilibrium in an attempt to compensate for the change of coordinates, caused by the error. The error value reaches tens of meters, which is unacceptable on ultra low altitudes. Therefore, the efficient usage of satellite navigation systems in the stabilization system (Figure 6a) is determined by topography and the mission and is only possible for altitudes greater than $2.5 \mathrm{~m}$ (Barbasov, 2012).

On the other hand, the efficiency of the optical stabilization system decreases with increasing altitude (Figure 6b), due to the limited spatial resolution of the final matrix of photosensitive cameras and limitations on focus range of optics used in it.

As a solution that combines the advantages of both systems and giving the ability to perform tasks of hovering over a given point, accurate take-off and landing, it is proposed to use a combined optical stabilization system based on the methods of motion compensation and stabilization system based on GPS (Figure 6c).

The decision on stabilization mode can be made on the following principles:

- on exceeding the altitude threshold on the barometric sensor (nominal change of stabilization mode),

- on ground command (forced change of stabilization mode),

- on flight plan,

- on inability of using onboard camera images due to weather conditions, lightning conditions or unsuitable horizontal surface (emergency change of stabilization mode).

\section{Conclusions}

This paper proposes a way to solve the problem of small sized UAV hovering over a given spatial point (maintaining of absolute spatial position) at a low altitude for a long time. The proposed method combines the advantages of existing systems and gives the ability to perform hovering over a given point, the exact take-off and landing. The main feature of method is the combination of optical stabilization system based on motion compensation methods using the computer vision, and stabilization system based on GPS. The limitations of implemented methods are determined and the algorithm is proposed to combine them in order to improve the efficiency.

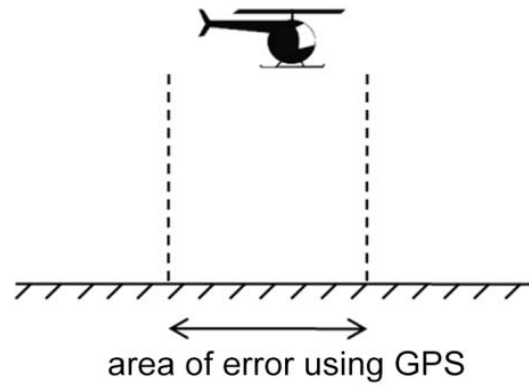

$a$

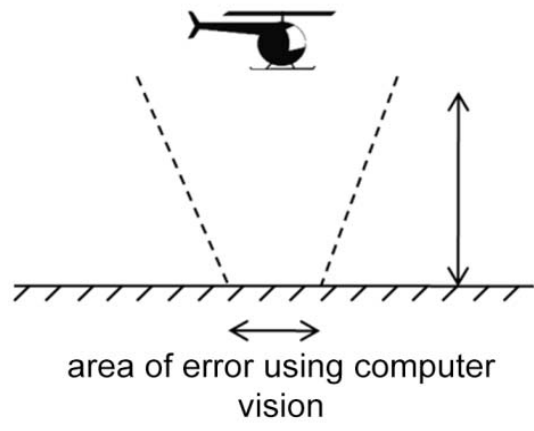

$b$

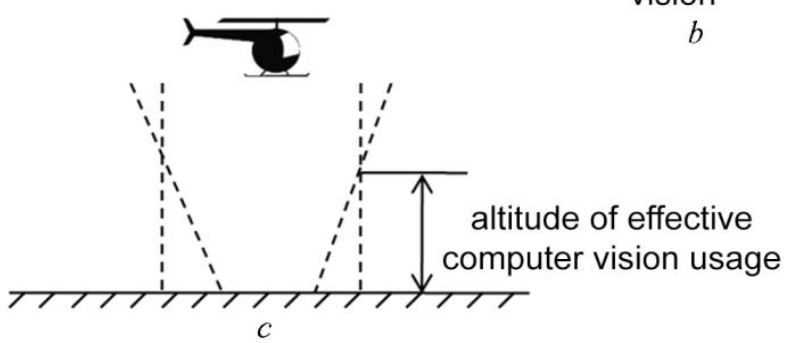

Figure 6. Usage of computer vision for UAV spatial positioning

\section{References}

1. Schmid, K., etc. (2012) View Planning for Multi-View Stereo 3D Reconstruction Using an Autonomous Multicopter. Journal of Intelligent \& Robotic Systems, 65(1-4), 309-323.

2. Barbasov, V.K., etc. (2012) Multirotor unmanned aerial vehicles and their capabilities for using in the field of earth remote sensing. Ingenernye Izyskaniya, 10, 38-42. (In Russian) 
3. Zinchenko, O.N. (2012) Unmanned aerial vehicles: the use of aerial photography in order to map, Part 1. Moscow: Racurs. 12 p. (In Russian)

4. DJI Innovations. (2013) NAZA for Multi-Rotor User Manual. Guangdong. (V 2.8 2013.05.03 Revision).

5. ETH IDSC. (2014) Flying Machine Arena. Zurich. Available online - http://www.idsc.ethz.ch.

6. Ritz, R., Muller, M.W., Hehn, M., D'Andrea, R. (2012) Cooperative quadrocopter ball throwing and catching. In Proceedings of Intelligent Robots and Systems (IROS), 2012 IEEE/RSJ International Conference, October 2012, (pp. 4972-4978). Vilamoura: IEEE.

7. Vatolin, D., Soldatov, S., Strelnikov, K. (2011) VirtualDub MSU Deshakerfilter. Moscow: MSU Graphics \& Media Lab.

8. Thalin, G. (2011) Deshaker - video stabilizer.www.guthspot.se. (3.0 ed).

9. Eckl, M.C., Snay, R.A., Soler, T., Cline, M.W., Mader, G.L. (2001) Accuracy of GPS-derived relative positions as a function of infestation distance and observing-session duration. Journal of Geodesy, 75, 633-640. 